\title{
Divergência genética entre acessos de pimentas com base em descritores morfológicos quantitativos
}

\author{
Genetic divergence between peppers accessions based on quantitative morphological \\ descriptors
}

\author{
Larissa Barbosa Silva \\ Leane Fialho de Melo \\ Verônica Brito da Silva \\ Ana Paula Peron \\ Ângela Celis de Almeida Lopes \\ Regina Lúcia Ferreira Gomes
}

Universidade Federal do Piauí - UFPI - Picos - Piauí - Brasil

\section{Resumo}

No presente trabalho analisou-se a divergência genética entre dez acessos de Capsicum $s p$, provenientes do Banco Ativo de Germoplasma da Universidade Federal do Piauí com base em descritores morfológicos quantitativos considerados relevantes para produtividade e para a ornamentação. Tal análise foi realizada por meio dos métodos de agrupamento de Tocher e hierárquico (UPMGA), com o emprego da distância de Malahobis como medida de dissimilaridade. A partir dos resultados obtidos verificou-se divergência significativa entre os acessos estudados, sendo o acesso BAGC 26, da espécie $C$. baccatum, o mais dissimilar em relação aos acessos BAGC 40 e BAGC 59 , da espécie $C$. annuum. Os descritores de maior contribuição para divergência genética entre as plantas de pimentas analisadas foram largura do fruto, comprimento do fruto e peso do fruto. Apesar do banco de germoplasma da Universidade Federal do Piauí (UFPI) ser recente e ainda contar com um pequeno número de acessos, os dados obtidos demonstram importante variabilidade genética entre os materiais analisados, condição que, mesmo preliminar, favorece a implantação de programa de melhoramento de pimentas no estado do Piauí.

\section{Abstract}

In this paper we analyzed the genetic diversity of ten accessions of Capsicum $s p$ from the Active Germplasm Bank of Federal University of Piauí based on quantitative morphological descriptors relevant to productivity, as well as for ornamental environments peppers. This analysis was performed using the Tocher clustering methods, hierarchical (UPMGA), with the use of Malahobis away as dissimilarity measure. From the results showed a significant divergence among accessions, and the BAGC 26 access, the species $C$. baccatum, the most dissimilar in relation to BAGC BAGC 40 and 59 , the species $C$. annuum. The greatest contribution descriptors for genetic divergence of the plants analyzed peppers were wide of fruit, fruit length and weight of the fruit. Despite the germplasm bank of Federal University of Piauí (UFPI) be recent and still have a small number of hits, the data here show significant genetic variability among the materials analyzed, a condition that, although preliminary, favors the implementation of peppers breeding program in the state Piaui.

\section{Palavras-chave}

Capsicum sp. Banco de germoplasma. Caracterização morfológica. Dissimilaridade genética.

\section{Keywords}

Capsicum sp; Germoplasm bank. Morphological characteristics. Dissimilarity genetic. 


\section{Introdução}

As pimentas Capsicum spp pertencentes a Família Solanaceae se destacam como um importante segmento do mercado de hortaliças frescas no Brasil, não somente pela alta rentabilidade, mas também por empregar elevada mão de obra no setor agrícola e comercial (HAVERROTH e NEGREIROS, 2011). Em média, 12.000 hectares de área são cultivadas no país por ano com pimentas e pimentões, envolvendo recursos de mais de 1,5 milhões de dólares somente na comercialização das sementes (NEITZKE et al., 2010; RODRIGUES et al., 2015). No entanto, diferentemente de outros estados brasileiros, no Piauí os dados sobre a produção de pimentas são imprecisos e irregulares e o cultivo dessas hortaliças é realizado apenas por pequenos agricultores (RODRIGUES et al., 2015). Ainda, grande parte das pimentas consumidas e/ou utilizadas para ornamentação no estado é importada de outras localidades do país, como de Minas Gerais, São Paulo, Ceará e Bahia (COSTA et al., 2006; RODRIGUES et al., 2015).

Dentre as espécies de pimentas, a $C$. annuum L. (pimentão), $C$. chinense Jaqc. (pimentade-cheiro), $C$. frutencens $L$. (pimentamalagueta) e $C$. baccatum $L$. (pimenta-dedo-de-moça) são as mais utilizadas pela população brasileira na culinária, medicina popular e ornamentação de ambientes (HAVERROTH e NEGREIROS, 2011). Tais espécies têm ampla variabilidade genética evidenciada, por exemplo, nas formas, tamanhos e colorações de frutos e flores (NEITZKE et al., 2010; MELO et al., 2014). Esta diversidade, considerada por muitos pesquisadores ainda como pouco explorada do ponto de vista econômico (KIM et al., 2011; SILVA NETO et al., 2014), combinada a excelente adaptação destas plantas ao clima tropical, fazem do Brasil um importante centro de diversidade de pimentas Capsicum, o que favorece a implantação de programas de melhoramento genético do gênero no país (HAVERROTH e NEGREIROS, 2011; VILLELA et al., 2014).

Todo programa de melhoramento é dependente da amplitude genética disponível em acessos presentes em bancos de germoplasma (SIGNORINI et al., 2013). Assim, esse material necessita ser bem conhecido e monitorado, já que a falta ou a inadequação de informações dificulta a utilização satisfatória do material conservado pelos melhoristas (NEITZKE et al., 2010). O estudo de divergência genética realizado por meio da dissimilaridade é uma importante ferramenta para o conhecimento da variabilidade entre indivíduos presentes nestes bancos (VASCONCELOS; BARBIERI, 2014), podendo ser calculada eficientemente por métodos de agrupamentos que separam de forma efetiva os acessos em grupos e permitem a verificação da máxima homogeneidade entre os indivíduos e a máxima heterogeneidade entre os grupos (SOUSA et al., 2012).

A formação destes agrupamentos representa uma importante informação na seleção de genitores dentro dos programas de melhoramento, pois a formação de novas populações podem ser baseadas na amplitude de suas distâncias genéticas e na potencialidade dos genitores em gerar híbridos com maior efeito heterótico e que proporcionem maior segregação em recombinações para fins de produção e, consequentemente, comerciais (SUDRÉ et al., 2005; VILLELA et al., 2014). Nesse contexto, objetivou-se nesse estudo avaliar a divergência genética por meio da dissimilaridade genética com base em dois métodos de agrupamento, entre 10 acessos de pimentas Capsicum oriundos do Banco Ativo de Germoplasma de Capsicum da Universidade Federal do Piauí a partir de 12 descritores morfológicos quantitativos de grande relevância para a cultura. 


\section{Material e métodos}

Este estudo foi desenvolvido no Departamento de Fitotecnia do Centro de Ciências Agrárias da Universidade Federal do Piauí (UFPI), no período de agosto de 2007 a novembro de 2009, em casa de vegetação, no município de Teresina - Piauí, situado a 0505'S de latitude, 42ํำ $\mathrm{W}$ de longitude e 72,70 m de altitude, cujo clima, pela classificação de Köppen, é do tipo $\mathrm{AS}^{\prime}$. Os dez acessos de pimenta utilizados são procedentes do Banco Ativo de Germoplasma de Capsicum da UFPI (Tabela 1), cujos códigos foram designados pela sigla BAGC seguidos do número referente a entrada do material na coleção.

Tabela 1. Relação dos dez acessos de Capsicum spp. utilizados no estudo de divergência genética, provenientes do Banco Ativo de Germoplasma de Capsicum sp. da Universidade Federal do Piauí. Teresina - PI.

\begin{tabular}{lcr}
\hline Acessos & Nome comum & Espécie \\
\hline BAGC 06 & Murici & Capsicum chinense \\
BAGC 07 & Peito-de-moça & Capsicum chinense \\
BAGC 11 & Peito-de-moça & Capsicum annuum var. glabriusculum \\
BAGC 23 & Olho-de-peixe & Capsicum chinense \\
BAGC 24 & Dedo-de-moça & Capsicum bacatum \\
BAGC 26 & Desconhecido & Capsicum baccatum var. pendulum \\
BAGC 36 & Pimenta-de-mesa & Capsicum annuum var. glabriusculum \\
BAGC 40 & Mexicana longa & Capsicum annuum var.annuum \\
BAGC 59 & Desconhecido & Capsicum annuum var. glabriusculum \\
BAGC 67 & Desconhecido & Capsicum annuum var. glabriusculum \\
\hline
\end{tabular}

Considerando a ordem apresentada na Tabela 1, os acessos BAGC 06 a BAGC 40 foram obtidos no estado do Piauí, enquanto que os BAGC 59 e BAGC 67 foram adquiridos no estado do Maranhão. É importante mencionar que o banco de germoplama de Capsicum da UFPI ainda possui um pequeno número de acessos, condição que justifica o número de acessos utilizados neste estudo.

No mês de setembro de 2007, as sementes dos acessos selecionados foram semeadas em copos de plástico descartáveis de $125 \mathrm{ml}$ contendo substrato Plantmax ${ }^{\circledast}$. As mudas, quando apresentaram seis folhas definitivas, em média, foram transplantadas para vasos plásticos de 4,5 litros, que continham uma mistura de terra vegetal e humus de minhoca, na proporção de 1:1. Para o tutoramento destes acessos, utilizou-se uma haste de madeira em cada vaso. A adubação foliar foi realizada com Ouro verde $(3 \mathrm{~g} / \mathrm{L})$, a cada quinze dias. A irrigação foi manual, realizada com um regador e realizada diariamente.

$O$ experimento obedeceu ao delineamento experimental inteiramente casualizado, com três repetições, sendo a parcela constituída por quatro vasos, com uma planta cada. Realizouse a identificação botânica dos dez acessos e, em seguida, preparou-se exsicatas, que posteriormente foram incorporadas ao Herbário Graziela Barroso da UFPI, com dois exemplares floridos de cada planta. A caracterização morfológica baseou-se em 12 descritores quantitativos estabelecidos pelo Internacional Plant Genetic Resources Institute, atualmente Bioversity Internacional, para o gênero Capsicum (IPGRI, 1995), que foram: altura da planta, largura da planta, comprimento do caule, diâmetro do caule, comprimento da folha, largura da folha, comprimento do fruto, largura do fruto, peso do fruto, espessura da parede do fruto, 
comprimento do pedicelo e persistência do fruto maduro. Tais caracteres são considerados primordiais na seleção de cultivares para fins comerciais. Para análise da divergência genética entre as plantas de pimentas, utilizou-se os métodos de agrupamentos de otimização de Tocher (RAO, 1952) e hierárquico UPGMA (Método não-ponderado de agrupamento aos pares), com o emprego da distância de Mahalanobis, como medida de dissimilaridade.

\section{Resultados e discussões}

Com base nos resultados observados Tabela 2, verifica-se que os acessos diferiram entre si em todos os caracteres avaliados, com exceção do diâmetro do caule que apresentou-se monomórfico. Para altura da planta, os valores médios variaram de 19,75 cm (BAGC 11) a $97,08 \mathrm{~cm}$ (BAGC 24), e para largura da planta, de $27,27 \mathrm{~cm}$ (BAGC 36) a 59,50 cm (BAGC 24), as médias gerais foram de $48,82 \mathrm{~cm}$ e $41,48 \mathrm{~cm}$, respectivamente. Quanto ao comprimento do caule, a variação observada foi de 6,42 cm (BAGC 11) a 43,03 cm (BAGC 23), com média geral de $20,61 \mathrm{~cm}$. O comprimento e a largura da folha apresentaram variação de 4,83 cm (BAGC 67) a 9,58 cm (BAGC 06) e 2,17 cm (BAGC 67) a 5,43 cm (BAGC 06), com médias gerais de 7,12 cm e $3,64 \mathrm{~cm}$, respectivamente.

Tabela 2. Médias referentes a dez acessos de Capsicum provenientes do Banco Ativo de Germoplasma de Capsicum da Universidade Federal do Piauí. Teresina - PI, em relação a doze caracteres morfológicos quantitativos, que foram AP = Altura da Planta, LP = Largura da Planta, $\mathrm{CC}=$ Comprimento do Caule, $\mathrm{DC}=$ Diâmetro do Caule, $\mathrm{CFO}=$ Comprimento da Folha, LFO = Largura da Folha, CFR = Comprimento do Fruto, LFR = Largura do Fruto, PFR = Peso do Fruto, EPFR = Espessura da Parede do Fruto, $\mathrm{CPED}=$ Comprimento do Pedicelo, PEFR = Persistência do Fruto Maduro.

\begin{tabular}{|c|c|c|c|c|c|c|c|c|c|c|c|c|}
\hline ACESSOS & $\begin{array}{c}\text { AP } \\
(\mathrm{cm})\end{array}$ & $\begin{array}{c}\text { DP } \\
(\mathrm{cm})\end{array}$ & $\begin{array}{c}\mathrm{CC} \\
\text { (cm) }\end{array}$ & $\begin{array}{c}\mathrm{DC} \\
(\mathrm{cm})\end{array}$ & $\begin{array}{c}\text { CFOM } \\
(\mathrm{cm})\end{array}$ & $\begin{array}{l}\text { LFOM } \\
\text { (cm) }\end{array}$ & $\begin{array}{c}\mathrm{CF} \\
(\mathrm{cm})\end{array}$ & $\begin{array}{c}\mathrm{LF} \\
(\mathrm{cm})\end{array}$ & $\begin{array}{c}\mathrm{PF} \\
(\mathrm{cm})\end{array}$ & $\begin{array}{c}\text { EP } \\
(\mathrm{mm})\end{array}$ & $\begin{array}{c}\mathrm{CP} \\
(\mathrm{cm})\end{array}$ & $\begin{array}{c}\text { PFM } \\
\text { (dias) }\end{array}$ \\
\hline 06 & $59,75 b$ & $47,83 b c$ & $26,50 \mathrm{bc}$ & $0,72 a$ & $9,58 a$ & $5,43 a$ & $1,45 \mathrm{de}$ & $1,61 \mathrm{c}$ & $1,53 \mathrm{~cd}$ & $1,67 \mathrm{bcd}$ & $2,26 b$ & $49,08 a b$ \\
\hline 07 & $42,17 \mathrm{c}$ & $48,08 \mathrm{bc}$ & $12,75 \mathrm{de}$ & $0,86 a$ & $7,07 b$ & $4,25 a b$ & $2,28 b c$ & $1,44 c$ & $1,65 \mathrm{~cd}$ & $2,09 a b$ & $1,65 c$ & $27,67 b$ \\
\hline 11 & $19,75 d$ & $31,08 d$ & $6,42 \mathrm{e}$ & $0,61 a$ & $5,40 \mathrm{bc}$ & $2,23 c$ & $2,52 b$ & $1,17 d$ & $1,81 \mathrm{~cd}$ & $1,74 b c$ & $1,97 b c$ & $35,00 a b$ \\
\hline 23 & $61,28 b$ & $42,89 c$ & $43,03 a$ & $0,87 a$ & $9,07 a$ & 4,41a & $0,96 \mathrm{e}$ & $0,86 \mathrm{ef}$ & $0,41 d$ & 1,44 cde & $2,01 b c$ & $44,08 a b$ \\
\hline 24 & $97,08 a$ & $59,50 a$ & $38,08 \mathrm{ab}$ & $0,86 a$ & $9,70 a$ & $4,96 a$ & $2,56 b$ & $1,94 b$ & $3,72 b$ & $2,54 \underline{a}$ & $2,13 b c$ & $48,33 a b$ \\
\hline 26 & $63,83 b$ & $54,89 a b$ & $23,64 \mathrm{~cd}$ & $0,73 a$ & $9,52 a$ & $5,36 a$ & $2,69 \mathrm{~b}$ & $4,12 a$ & $9,35 a$ & $2,48 a$ & $3,39 a$ & $47,67 a b$ \\
\hline 36 & $26,25 \mathrm{~cd}$ & $27,27 d$ & $8,58 \mathrm{e}$ & $0,50 a$ & $4,88 c$ & $2,58 c$ & $1,33 \mathrm{de}$ & $1,07 \mathrm{de}$ & $0,87 d$ & 1,51bcde & $1,83 b c$ & $58,83 a$ \\
\hline 40 & $70,31 b$ & $43,75 c$ & $25,25 c$ & $0,73 a$ & $6,12 b c$ & $2,74 b c$ & $9,65 a$ & $0,64 f$ & $2,59 b c$ & $1,04 \mathrm{e}$ & $2,93 a$ & $39,36 a b$ \\
\hline 59 & $26,25 \mathrm{~cd}$ & $29,83 d$ & $10,50 \mathrm{e}$ & $0,50 a$ & $5,01 \mathrm{c}$ & $2,22 c$ & $1,73 \mathrm{~cd}$ & $0,65 f$ & $0,56 d$ & 1,10de & $2,02 b c$ & $54,50 a b$ \\
\hline 67 & $31,50 \mathrm{~cd}$ & $29,66 d$ & $11,33 \mathrm{e}$ & $0,62 a$ & $4,83 c$ & $2,17 \mathrm{c}$ & $1,62 d$ & $0,77 f$ & $0,77 d$ & $1,17 \mathrm{cde}$ & $1,80 \mathrm{bc}$ & $61,67 a$ \\
\hline MG & 49,82 & 41,48 & 20,61 & 0,70 & 7,12 & 3,64 & 2,68 & 1,43 & 2,33 & 1,68 & 2,19 & 46,62 \\
\hline CV (\%) & 11,17 & 8,84 & 19,65 & 20,98 & 9,31 & 14,92 & 7,35 & 5,88 & 23,99 & 12,08 & 8,29 & 22,61 \\
\hline
\end{tabular}

Ainda na Tabela 2, com relação ao comprimento do fruto, a variação foi de $0,96 \mathrm{~cm}$ (BAGC 23) a 9,65 cm (BAGC 40); largura do fruto, de 0,64 cm (BAGC 40) a 4,12 cm (BAGC 26) e peso do fruto, de 0,41 g (BAGC 23) a 9,35 g (BAGC 26), com médias gerais de 2,68 cm, 1,43 cm e 2,33 cm, respectivamente. Para espessura da parede do fruto, as médias variaram de 1,04 $\mathrm{cm}$ (BAGC 40) a 2,54 (BAGC 24), com média geral de 1,68 cm. O comprimento do pedicelo apresentou médias de 1,65 cm (BAGC 07) a 3,39 cm (BAGC 26). Já a persistência do fruto 
maduro na planta apresentou valores médios variando de 27,67 dias (BAGC 07) a 61,67 dias (BAGC 67), com média de 46,62 dias.

Esses resultados evidenciam a existência de importante variabilidade genética entre acessos de Capsicum do BAGC da UFPI, condição esta já relatada em estudos desenvolvidos por Monteiro et al. (2010), Neitzke et al. (2010), Haverroth \& Negreiros (2011) e Melo et al. (2014), que avaliaram a divergência genética entre as espécies $C$. annuum, $C$. chinense, e $C$. baccatum e observaram ampla variabilidade em caracteres morfológicos quantitativos relacionados a caule, folha e fruto destas plantas.

$\mathrm{Na}$ Tabela 3 as medidas de dissimilaridade genética, estimadas pela distância generalizada de Mahalanobis entre os dez acessos (Tabela 3), também mostraram que os menores valores de dissimilaridade foram entre BAGC 59 e BAGC 67 (14,65), da espécie $C$. annuumm. Logo, foram os mais similares. Entre os acessos da espécie $C$. chinense, os menores valores de dissimilaridade foram entre BAGC 06 e BAGC $07(97,58)$. Já os maiores valores ocorreram entre BAGC 26 (C. baccatum) e BAGC 40 (C. annuum) (11027, 36); BAGC 26 (C. baccatum) e BAGC 59 (C. annuum) $(5466,64)$. Esses acessos com as maiores distâncias de dissimilaridade representam boa oportunidade de ganho heterótico quando cruzados entre si, com a possibilidade de produzir genótipos superiores em gerações segregantes. Esses resultados estão em sintonia com os dados médios descritos entre os acessos para onze dos doze caracteres heteromórficos avaliados, tendo em vista que os maiores valores obtidos em cada caráter quase sempre foram para os acessos BAGC 59, BAGC 26 e BAGC 40.

Tabela 3. Distância generalizada de Mahalanobis entre dez acessos de pimentas do Banco Ativo de Germoplasma de Capsicum (BGC) da Universidade Federal do Piauí.

\begin{tabular}{|c|c|c|c|c|c|c|c|c|c|c|}
\hline ACESSOS & $\begin{array}{c}\text { BAGC } \\
06\end{array}$ & $\begin{array}{c}\text { BAC } \\
07\end{array}$ & $\begin{array}{c}\text { BAGC } \\
11\end{array}$ & $\begin{array}{c}\text { BAGC } \\
23\end{array}$ & $\begin{array}{c}\text { BAGC } \\
24\end{array}$ & $\begin{array}{c}\text { BAGC } \\
26\end{array}$ & $\begin{array}{c}\text { BAGC } \\
36\end{array}$ & $\begin{array}{c}\text { BAGC } \\
40\end{array}$ & $\begin{array}{c}\text { BAGC } \\
59\end{array}$ & $\begin{array}{c}\text { BAGC } \\
67\end{array}$ \\
\hline BAGC 06 & 0,00 & 97,58 & 236,69 & 526,31 & 200,07 & 2674,52 & 362,29 & 4721,86 & 683,39 & 623,42 \\
\hline BAGC 07 & & 0,00 & 182,79 & 741,76 & 230,95 & 2621,54 & 429,84 & 4492,06 & 770,66 & 707,61 \\
\hline BAGC 11 & & & 0,00 & 478,66 & 560,18 & 3444,93 & 102,93 & 4316,60 & 282,86 & 269,26 \\
\hline BAGC 23 & & & & 0,00 & 753,95 & 5429,60 & 331,07 & 4023,02 & 188,46 & 172,66 \\
\hline BAGC 24 & & & & & 0,00 & 2992,01 & 813,02 & 3815,04 & 1077,57 & 979,36 \\
\hline BAGC 26 & & & & & & 0,00 & 4042,94 & $.1027,36$ & 5466,64 & 5322,59 \\
\hline BAGC 36 & & & & & & & 0,00 & 4974,18 & 143,50 & 119,58 \\
\hline BAGC 40 & & & & & & & & 0,00 & 4054,73 & 4177,83 \\
\hline BAGC 59 & & & & & & & & & 0,00 & 14,65 \\
\hline BAGC 67 & & & & & & & & & & 0,00 \\
\hline
\end{tabular}

BAGC: Banco ativo de germoplasma de Capsicum

As análises de agrupamento dos acessos obtidas pelos métodos de agrupamento de Tocher e hierárquico UPGMA, com base nos 12 descritores morfológicos quantitativos, resultaram na formação de três grupos distintos como pode ser visto na tabela 04 e na Figura 01. 
Tabela 4. Agrupamento de dez acessos de pimenta Capsicum sp provenientes do Banco Ativos de Germoplasma da Universidade Federal do Piauí, pelo método de otimização de Tocher, com base em 12 descritores morfológicos quantitativos, utilizando a distância generalizada de Mahalanobis. Teresina, PI, 2009.

\begin{tabular}{cl}
\hline Grupo & Acesso \\
\hline I & BAGC 59, BGC 67, BGC 36, BGC 11, BGC 23, BGC 06, BGC 07, BGC 24 \\
II & BAGC 26 \\
III & BAGC 40 \\
\hline
\end{tabular}

BAGC: Banco ativo de germoplasma de Capsicum.

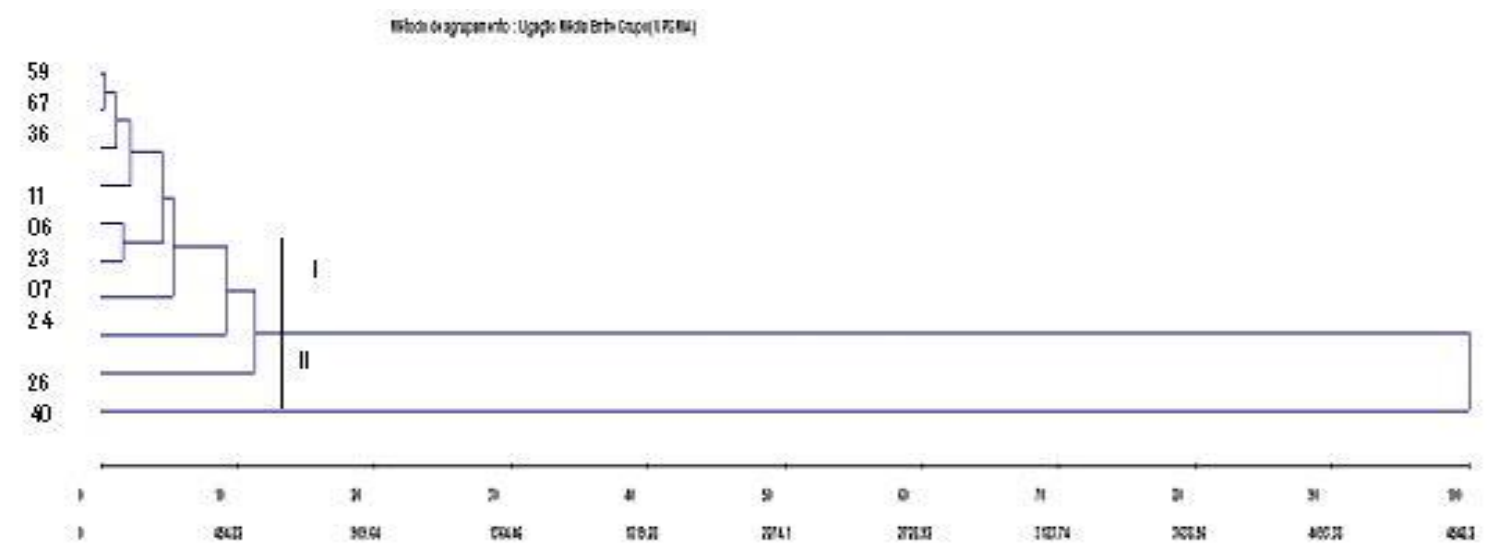

Figura 1. Dendograma da análise de agrupamento de dez acessos de pimentas do gênero Capsicum pelo método UPGMA, utilizando a distância de Mahalanobis, com base em doze descritores morfológicos quantitativos. Teresina, PI, 2009.

Baseado na Tabela 04 e na Figura 01, observa-se que o primeiro grupo concentrou os acessos BAGC 59, BAGC 67, BAGC 36, BAGC 11, BAGC 23, BAGC 06, BAGC 07 e BAGC 24 pertencentes as espécies $C$. chinense e $C$. annuum, que se agruparam por apresentar valores médios para maioria dos descritores; o segundo e o terceiro contiveram exclusivamente os acessos BAGC 26 (C. baccatum) e BAGC 40 (C. annuum), respectivamente. O BAGC 26 foi um dos que apresentou as médias mais altas para os descritores largura do fruto, peso do fruto e espessura da parede do fruto. Já o BAGC 40 apresentou a maior média para comprimento do fruto e as menores para largura do fruto e espessura da parede do fruto. Verifica-se que os acessos com os menores valores de dissimilaridade (BAGC 59 e BAGC 67; BAGC 06 e BAGC 07) foram alocados no grupo I. Já os acessos que apresentaram os maiores valores de dissimilaridade (BAGC 26 e BAGC 40; BAGC 26 e BAGC 59) estão inseridos em grupos distintos (II12 e III; II e I, respectivamente).

Resultado semelhante foi descrito por Monteiro et al. (2010), que ao analisarem a divergência genética entre acessos de pimentas Capsicum, por meio da técnica molecular RAPD, verificaram que os acessos das espécies $C$. annuum e $C$. chinense foram alocados em grupos distintos. Os autores inferiram que entre essas duas espécies de pimentas existe uma maior dissimilaridade genética, o que possibilita, de forma mais efetiva, a troca de genes entre elas. Os grupos formados pela dispersão gráfica são importantes por auxiliar na seleção de genótipos divergentes que serão utilizados em cruzamentos artificiais (NEITZKE et al., 2010). 
Os resultados com RAPD confirmam a importância dos marcadores morfológicos na determinação de divergência entre os acessos de pimentas.

A divergência genética observada entre o BAGC 26 (C. baccatum) em relação aos outros acessos é explicada pela ampla diferença morfológica evidenciada neste acesso em relação as outras espécies de pimentas, com grande ênfase ao tamanho e forma de suas folhas e frutos (CARVALHO \& BIANCHETTI, 2004). Já a divergência genética observada na Tabela 2, entre os acessos de $C$. annuum do grupo I em relação ao acesso de $C$. annuum do grupo III, é entendida em função desta espécie possuir diferentes variedades que divergem de forma significativa entre si, mostrando, por exemplo, variações de altura da planta, tamanho e diâmetro dos frutos e folhas (PERRY et al., 2007).

Portanto, é possível concluir a partir das análises realizadas que o acesso BAGC 26, da espécie $C$. baccatum, é o mais dissimilar em relação a BAGC 40 e BAGC 59, da espécie $C$. annuum, e de que os descritores com maior contribuição para divergência genética entre os acessos de pimentas analisados foram largura do fruto, comprimento do fruto e peso do fruto. Tais resultados podem contribuir para o direcionamento dos cruzamentos em programas de melhoramento de Capsicum, com o desenvolvimento de cultivares de maior interesse econômico. Ademais, espera-se que tais resultados, ainda que iniciais, estimulem a implantação no Piauí de um programa de melhoramento de pimentas, uma vez que o cultivo dessa hortaliça no estado é feito exclusivamente por pequeno produtores que comercializam sua produção apenas com feiras e pequenos supermercados.

\section{Referências}

1. BATISTA, M.R.A.; SILVA-FILHO, D.F. Caracterização morfoagronômica de pimentas não pungentes do gênero Capsicum spp., da Amazônia. Revista Agro@mbiente On-line, 89, p. 204-211, 2014.

2. CARVALHO, S.I.C.; BIANCHETTI, L.B. Sistema de produção de pimentas (Capsicum spp.): botânica. Embrapa Hortaliças, Brasília. 2004. Disponível em: <http://www. cnph. embrapa. sisprod/pimenta/index.htm>Acesso em 22 nov. 2016>.

3. COSTA, F.R; PEREIRA, T.N.S.; VITÓRIA, A.P.; CAMPOS, K.P., RODRIGUES, R.; SILVA, D.H., PEREIRA, M.G. Genetic diversity among Capsicum accessions using RAPD markers. Crop Breeding and Applied Biotechnology, 6, p. 18-23, 2006.

4. HAVERROTH, M.; NEgREIROS, P.R.M. Calendário agrícola, agrobiodiversidade e distribuição espacial de roçados Kulina (Madeja), Alto Rio Envira, Acre, Brasil. Sitientibus série Ciências Biológicas, 11, p. 299-308, 2011.

5. IPGRI. Descritores para Capsicum (Capsicum spp). Roma: IGRI, 1995. 51 p.

6. KIM, J.S.; AHN, J.; LEE, S.J.; MOON, B.; HA, T.Y.; KIM, S. Phytochemicals and antioxidant activity of fruits and leaves of paprika (Capsicum annuum L., var. special) cultivated in Korea. Journal of Food Science, 76, p. 193-198, 2011. 
7. MELO, L.F.; GOMES, R.L.F.; SILVA, V.B.; MONTEIRO, E.R.; LOPES, A.C.A.; PERON, A.P. Potencial ornamental de acessos de pimenta. Ciência Rural, 44, p. 2010-2015, 2014.

8. MONTEIRO, E. R., BASTOS, E. M., LOPES, A.C.D. A., GOMES, R.L.F., NUNES, J.A.R. Genetic diversity among accessions of cultivated peppers species. Ciência Rural, 40, p. 258-263, 2014.

9. NEITZKE, R.S.; BARBIERI, R.L.; RODRIGUES, W.F.; CORRÊA, I.V.; CARVALHO, F.I.F. Dissimilaridade genética entre acessos de pimenta com potencial ornamental. Horticultura Brasileira, 28, p. 47-53, 2014.

10. PERRY, L.; DICKAU, R.; ZARRILLO, S.; HOLST, I.; PEARSALL, D.M.; PIPERNO, D.R.; ZEIDLER, J.A. Starch fossils and the domestication and dispersal of chili peppers (Capsicum spp. L.) in the Americas. Science, 315, p. 986-988, 2007.

11. RAO, R.C. Advanced statistical methods in biometric research. Nova York: J. Wiley, 1952. $330 \mathrm{p}$.

12. SIGNORINI, T.; RENESTO, E.; MACHADO, M.F.P.S., DAS, N., BESPALHOK, D.; MONTEIRO, E.R. Diversidade genética de espécies de Capsicum com base em dados de isozimas. Horticultura Brasileira, 31, p. $534-539,2013$.

13. SILVA-NETO, J.J.D.; RÊGO, E.R.D.; NASCIMENTO, M.F.; SILVA-FILHO, V.A.L.; ALMEIDANETO, J.X.D.; RÊGO, M.M.D. Variability in a base population of pepper (Capsicum annuum L.). Revista Ceres, 61, p. 84-89, 2014.

14. SINGH, D. The relative importance of characters affecting genetic divergence. The Indian Journal of Genetic and Plant Breeding, v. 41, p. 237-245, 1981.

15. SOUSA, L.B.D.E.; SILVA, E.M.; GOMES, R.L.F.; LOPES, A.C.D.A.; VERAS, I.C.S. Caracterização e divergência genética de acessos de Passiflora edulis e $P$. cincinnata com base em características físicas e químicas de frutos. Revista Brasileira de Fruticultura, 34, p. 832-839, 2012.

16. SUDRÉ, C.P.; RODRIGUES, R.; RIVA, E.M.; KARASAWA, M., AMARAL-JÚNIOR, A.T. Divergência genética entre acessos de pimentas e pimentões utilizando técnicas multivariadas. Horticultura Brasileira, 23, p. 22-27, 2005.

17. VASCONCELOS, C.S.; BARBIERI, R.L.; NEITZKE, R.S.; PRIORI, D.; FISCHER, S.Z.; MISTURA, C.C. Determinação da dissimilaridade genética entre acessos de Capsicum chinense com base em características de flores. Revista Ceres, 59, p. 493-498, 2012.

18. VILLELA, J.C.; BARBIERI, R.L.; CASTRO, C.M.; NEITZKE, R.S.; VASCONCELOS, C.S.; CARBONARI, T., PRIORI, D. Molecular characterization of landraces of peppers (Capsicum baccatum) with SSR markers. Horticultura Brasileira, 32, p. 131-137, 2014. 